\title{
Effective Description of Critical QCD
}

\section{N. G. Antoniou*}

Department of Physics, University of Athens, GR-15784, Athens, Greece

E-mail: nantonio@phys.uoa.gr

\section{F. K. Diakonos}

Department of Physics, University of Athens, GR-15784, Athens, Greece

E-mail: fdiakono@phys.uoa.gr

Employing universality arguments, we construct, based on the critical $3 \mathrm{~d}$ Ising effective action [M. M. Tsypin, Phys. Rev. Lett. 73, 2015 (1994)], the Ising-QCD partition function for the description of the net baryon density in the immediate neighbourhood of the QCD critical endpoint, remnant of the chiral phase transition. We demonstrate that the constructed partition function satisfies all the requirements of scaling theory and universality, not only for an infinite system, but also in the finite size scaling regime. Furthermore, we use the Ising -QCD partition function to obtain predictions relevant to measurements in the search for the QCD critical point in contemporary and future experiments with colliding nuclei.

Corfu Summer Institute 2018 "School and Workshops on Elementary Particle Physics and Gravity" (CORFU2018)

31 August - 28 September, 2018

Corfu, Greece

${ }^{*}$ Speaker. 


\section{Introduction}

The exploration of the phase diagram of strongly interacting matter has become the focus of intensive theoretical and experimental efforts during the last decades. The current theoretical understanding of the phases of QCD matter is mainly based on universality arguments, effective models and Lattice QCD calculations. The later predict a smooth crossover from the hadronic to the quark-gluon phase at vanishing baryochemical potential $\left(\mu_{b}=0\right)[1]$ and high temperatures $T$. On the other hand phenomenological arguments based on effective models predict a first order transition for large baryonic densities and low temperatures [2,3]. These two limiting scenarios suggest the presence of a second order transition from the hadron to the quark-gluon world at a critical point $\left(\mu_{c}, T_{c}\right)$, occurring as the endpoint of a line of first order transitions. This critical endpoint (CEP) should be considered as the remnant of the chiral critical point in massless QCD and its detection constitutes the holly grail for understanding strong interactions at finite temperature and density.

However, despite the intensive theoretical work on the QCD phase diagram there are no strict predictions, up to now, for the location and even the existence of this CEP. The reason is that the only available fundamental tool to deal with strongly interacting matter, the Lattice QCD, suffers from the so called "sign problem" at finite baryonic density $\left(\mu_{b}>0\right)$ and therefore its predictions in this regime are questionable. As a consequence the experimental exploration of the QCD phase diagram becomes of higher priority. Current and future ion collision experiments are planned to cover a wide range of this phase diagram: The crossover region (low $\mu_{b}$, high $T$ ) is probed by the LHC (CERN) and RHIC (BNL) experiments, while the baryon rich region characterized by first order transitions is investigated by FAIR (GSI) and NICA (forthcoming, JINR) experiments. Recently it is argued that gravitational waves emitted from neutron stars could also provide invaluable information concerning the equation of state and the first order transitions occurring in this regime. Finally, in the intermediate region the experiments NA49 and NA61/SHINE (CERN, SPS) as well as the RHIC-BES are expected to be able to approach the neighbourhood of the CEP.

In the experimental search for the QCD CEP the main task is to provide appropriate observables capturing the critical characteristics. In this effort, the guiding quantity is the order parameter and its fluctuations which are expected to be power-law distributed at the CEP. The natural order parameter for the remnant of the chiral transition is the chiral condensate which, due to its coupling to the nucleons, transfers the critical fluctuations to the net-baryon density, and in particular the proton density, as well [4]. As argued in recent works [5], factorial moment analysis of the proton density could reveal the approach to the CEP through the phenomenon of intermittency which is the manifestation of critical opalescence in sub-nuclear scales. Additionally, the formation of tails in the distribution of the order parameter fluctuations close to the CEP, is expected to lead to nonmonotonic behaviour of the associated higher order cumulants [6], a scenario explored intensively in RHIC-BES program.

The aim of the present work is to show that it is possible, employing the Ising-QCD partition function, which is constructed from first principles (universality class arguments), to get significant quantitative predictions for both, the appearance of the intermittency phenomenon in the neighbourhood of the CEP as well as the behaviour of the higher order cumulants within the critical region. 


\section{The order parameter(s) of critical QCD}

The thermodynamics close to the critical point is described by the fluctuations of the order parameter. For strongly interacting matter, the order parameter of the QCD critical point is the chiral condensate: $\langle\bar{\Psi} \Psi\rangle=\bar{u} u+\bar{d} d$ which defines the scalar-isoscalar field $\sigma=\langle\bar{\Psi} \Psi\rangle$, remnant from chiral phase transition. In fact, the tricritical point related to the spontaneous breaking of $O(4)$ symmetry, carried by the isovector $(\sigma, \vec{\pi})$ in massless QCD [7], becomes a 3d-Ising critical point describing the spontaneous breaking of $Z_{2}$-symmetry carried by the isoscalar field $\sigma$ in massive QCD [8].

In a finite-density medium the sigma field is coupled to the baryons. At leading order with respect to baryonic density $n_{b}$ and temperature $T$ one can write [3,9]:

$$
\sigma \approx\langle\bar{\Psi} \Psi\rangle_{0}-\frac{T^{2}}{8 f_{\pi}^{2}}\langle\bar{\Psi} \Psi\rangle_{0}-\frac{\Sigma_{\pi N}}{f_{\pi}^{2} m_{\pi}^{2}}\langle\bar{\Psi} \Psi\rangle_{0} n_{b}
$$

where $\langle\bar{\Psi} \Psi\rangle_{0}$ is the condensate at zero baryonic density and temperature. Thus, the fluctuations of the $\sigma$-field have the form:

$$
\delta \sigma \approx-\frac{\Sigma_{\pi N}}{f_{\pi}^{2} m_{\pi}^{2}}\langle\bar{\Psi} \Psi\rangle_{0} \delta n_{b} \quad ; \quad \delta \sigma \sim \delta n_{b}
$$

where $\delta \sigma$ are the fluctuations of the $\sigma$-field related to the corresponding isothermal susceptibility, while $\delta n_{b}$ are the baryon-density fluctuations. For $T \rightarrow T_{c}$ the isothermal susceptibility diverges (infinite system) and the fluctuations $\delta \sigma$ and $\delta n_{b}$ attain the same singular behaviour. This qualifies the net-baryon density $n_{b}$ to an equivalent order parameter for the QCD critical system, which being a conserved quantity:

$$
\frac{\partial n_{b}}{\partial t}+\overrightarrow{i k} \cdot \overrightarrow{J_{b}}(\vec{k}, t)=0
$$

dominates, as a slow variable, in the long-wavelength $(k \rightarrow 0)$ limit. In the following we will focus on the description of the baryon-density fluctuations close to the CEP.

\section{Ising-QCD partition function}

We employ the theoretical arguments supporting that the QCD CEP belongs to the 3d-Ising universality class to construct a partition function describing the thermodynamics of the QCD critical system. The critical state of the 3d-Ising model is uniquely described by the following set of critical exponents:

$$
\alpha \approx 0, \beta \approx \frac{1}{3}, \gamma \approx \frac{4}{3}, \delta \approx 5, v \approx \frac{2}{3}, \eta \approx 0
$$

The effective action capturing the equation of state for the critical 3d-Ising system is given as [10]:

$$
S_{e f f}=\int_{V} d^{3} \mathbf{x}\left[\frac{1}{2}|\nabla \phi|^{2}+U(\phi)-h \phi\right]
$$

with the effective potential:

$$
U(\phi)=\frac{1}{2} m^{2} \phi^{2}+m g_{4} \phi^{4}+g_{6} \phi^{6} ; m=\beta_{c} \xi^{-1}\left(g_{4} \approx 1, g_{6} \approx 2\right)
$$


where the order-parameter field $\phi$ is the net magnetization (number of spins up minus number of spins down) in a space cell of volume $\delta V$ (measured in units $\left.\beta_{c}^{3}\right): \phi=\beta_{c}^{3} \lim _{\delta V \rightarrow 0}\left(\frac{n_{\uparrow}-n_{\downarrow}}{\delta V}\right)$ and $\beta_{c}=\frac{1}{k_{B} T_{c}}$. In Eq. (3.3) $\xi$ is the correlation length and $g_{4}, g_{6}$ are dimensionless coupling constants. For an infinite system the correlation length $\xi$ depends on the temperature $T$ in a singular way as $T \rightarrow T_{c}$ :

$$
\xi \sim\left|1-\frac{T}{T_{c}}\right|^{-v}
$$

We can map the effective action of the 3d-Ising critical system to the effective action of the QCD critical point making use of the following Ising-QCD correspondence:

- $\left(n_{\uparrow}, n_{\downarrow}\right) \longrightarrow\left(N_{B}, N_{\bar{B}}\right)$ where $N_{B}$ and $N_{\bar{B}}$ are the baryon (spin up) and the antibaryon (spin down) numbers respectively.

- Consequently the magnetization density is mapped to the net-baryon density: $\phi \longrightarrow \beta_{c}^{3} n_{b}$

- Finally, the external magnetic field $h$ is mapped to the baryochemical potential (in units of $\left.\beta_{c}^{-1}\right): h \longrightarrow\left(\mu-\mu_{c}\right) \beta_{c}$.

With this mapping we obtain from Eq. (3.4) the critical QCD adapted effective action $S_{\text {eff,QCD }}\left[n_{b}\right]$ which leads to the Ising-QCD partition function:

$$
\mathscr{Z}_{I Q C D}=\sum_{\left\{n_{b}\right\}} \exp \left(-S_{\text {eff,QCD }}\left[n_{b}\right]\right)
$$

containing a sum over $n_{b}$-configurations. Since $n_{b}$ is the slow mode, the long wavelength (constant) configurations, for which $\nabla n_{b} \approx 0$, dominate in the sum in Eq. (3.5). This allows to express the partition function (3.5) in the grand-canonical description as [11]:

$$
\mathscr{Z}_{I Q C D}=\sum_{N_{b}=0}^{\Lambda} \zeta^{N_{b}} \exp \left[-\frac{1}{2} m^{2} \frac{N_{b}^{2}}{\Lambda}-m g_{4} \frac{N_{b}^{4}}{\Lambda^{3}}-g_{6} \frac{N_{b}^{6}}{\Lambda^{5}}\right]
$$

with $\Lambda=\beta_{c}^{-3} V$ ( $V$ being the volume), $m \sim|t|^{v}, \zeta=\exp \left(\frac{\mu-\mu_{c}}{T_{c}}\right)$ and $t=\frac{T-T_{c}}{T_{c}}$ the reduced temperature. Our claim is that $\mathscr{Z}_{I Q C D}$ in Eq. (3.6) describes correctly a critical system of protons, very close to the critical point $(\zeta \approx 1, t \approx 0)$.

\section{Scaling laws for the infinite system}

In this section we will demonstrate that the Ising-QCD partition function in Eq. (3.6) leads to the correct scaling laws characterizing the $3 \mathrm{~d}$-Ising universality class at the critical point. To this end we consider the general class of systems:

$$
\mathscr{Z}(\zeta, \Lambda, T)=\sum_{N=0}^{\Lambda} \zeta^{N} \exp [-\beta F(N, \Lambda, T)]
$$

with free-energy $F(N, \Lambda, T)$ obeying:

$$
F\left(N, \Lambda, T_{c}\right)=F_{c}\left(\frac{N}{\Lambda^{q}}\right) \quad ; \quad q<1, \Lambda \gg 1
$$




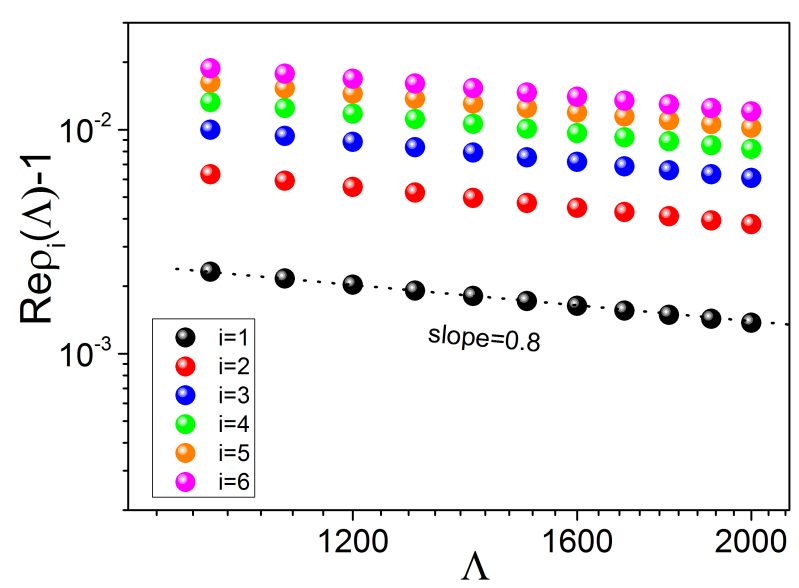

Figure 1: The real part of the zeroes of the partition function (4.1) with $q=\frac{5}{6}$ as a function of the system's size $\Lambda$ in $\log -\log$ scale. Only the first 6 zeroes of $\mathscr{Z}_{I Q C D}$ (nearest to $\zeta=(1,0)$ ) are displayed.

at the critical temperature $T_{c}$. The Ising-QCD partition function $\mathscr{Z}_{I Q C D}$ belongs to this class with $q=\frac{5}{6}$.

To explore the properties of the partition function $\mathscr{Z}$ in Eq. (4.1) at a more fundamental level we utilize the Lee-Yang theory of phase transitions [12]. In this treatment the zeroes $\left\{\rho_{i}(\Lambda, T)\right\}$ of the partition function in the complex $\zeta$-plane play a central role: At $T=T_{c}$ they accumulate at the critical point $\zeta=1$ for $\Lambda \rightarrow \infty$ (infinite system). The underlying accumulation law determines the scaling properties of the critical system.

In terms of $\left\{\rho_{i}(\Lambda, T)\right\}$ the partition function $\mathscr{Z}$ is represented as:

$$
\mathscr{Z}=\prod_{i=1}^{\Lambda}\left[\zeta-\rho_{i}(\Lambda, T)\right]
$$

Using this representation, it is straightforward to calculate the first multiplicity moment $\langle N\rangle$ :

$$
\langle N\rangle=\zeta \frac{\partial \ln \mathscr{Z}}{\partial \zeta} \quad ; \quad\langle N\rangle=\sum_{i=1}^{\Lambda} \frac{\zeta}{\zeta-\rho_{i}(\Lambda, T)}
$$

and in a similar manner all the higher moments $\left\langle N^{k}\right\rangle$. At the critical point these moments should obey scaling-laws with exponents related to $q$. The $q$-class partition function $\mathscr{Z}$ satisfies the requirements of scaling theory for $\Lambda \gg 1$ if:

$$
\rho_{i}\left(\Lambda, T_{c}\right)=1+\gamma_{i} \Lambda^{-q} \quad ; \quad \gamma_{i} \in \mathbb{C}
$$

This is an important property and its validity has been checked numerically for the $q$-class partition function setting $q=\frac{5}{6}$. The results for the 6 nearest to $\zeta=(1,0)$ zeroes, are shown in Figs. 1, 2 in double logarithmic scale. We observe the validity of the scaling law (4.5) with the correct exponent $q$ as $\Lambda \rightarrow \infty$. 


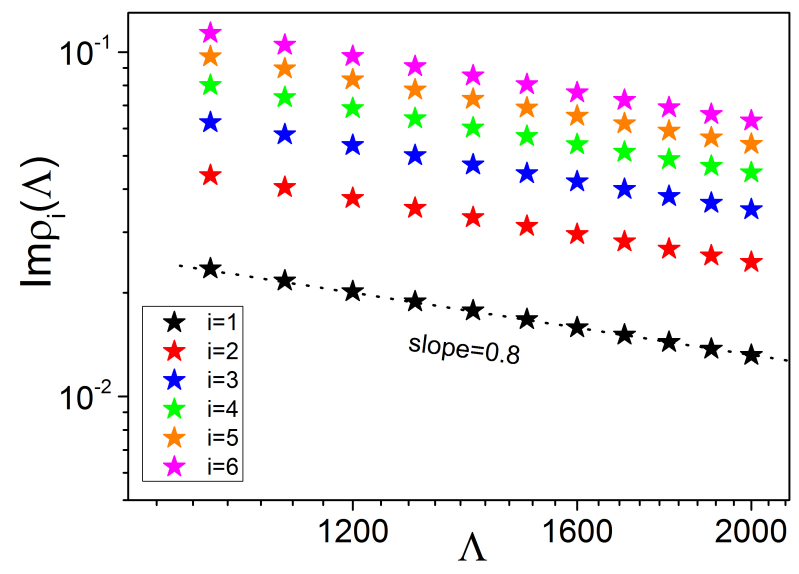

Figure 2: The imaginary part of the zeroes shown in Fig. 1 as a function of the system's size $\Lambda$ in $\log$-log scale.

In fact, using the scaling law in Eq. (4.5) one can derive a complete set of power-laws for the Ising-QCD theory in the limit $\Lambda \rightarrow \infty$ :

- Order parameter $\left(T \rightarrow T_{c}, \zeta \rightarrow 1\right)$ :

$$
\left\langle n_{b}\right\rangle \sim t^{v d(1-q)} \longrightarrow \beta=v d(1-q)
$$

- Order parameter, isothermal $\left(T=T_{c}, \zeta \rightarrow 1\right)$ :

$$
\left\langle n_{b}\right\rangle \sim(\zeta-1)^{\frac{1-q}{q}} \longrightarrow \delta=\frac{1-q}{q}
$$

- Susceptibility $\left(T \rightarrow T_{c}, \zeta=1\right)$ :

$$
\chi \sim t^{-v d(2 q-1)} \longrightarrow \gamma=v d(2 q-1)
$$

- Specific heat $\left(T \rightarrow T_{c}, \zeta=1\right)$ :

$$
c \sim t^{v d-2} \longrightarrow \alpha=v d-2
$$

- Correlation function for large distances $r\left(T \rightarrow T_{c}, \zeta \rightarrow 1\right)$ :

$$
\Gamma(r) \sim r^{-2 d(1-q)} \longrightarrow \eta=2+d-2 d q
$$

Thus, one obtains the standard solution of scaling theory for the critical exponents:

$$
\begin{gathered}
\alpha=2-v d \quad ; \quad \beta=v d(1-q) ; \gamma=v d(2 q-1) \\
\delta=\frac{q}{q-1} ; \eta=2+d-2 q d
\end{gathered}
$$

in terms of the two fundamental critical indices $q$ and $v$ which are incorporated in the Ising-QCD partition function. Consequently, the infinite system's description with Ising-QCD is fully compatible with scaling theory laws! 


\section{Finite-size scaling (FSS)}

The effective description of critical QCD, through the Ising -QCD partition function, satisfies the requirements of scaling theory, not only for the infinite system but also for a system of finite volume $\Lambda$ (finite-size scaling) at the critical point $(\zeta=1, t=0)$.

In fact, for a large but finite volume $\Lambda$, one obtains the power laws expected from scaling theory of second-order phase transitions in the FSS regime, if the correlation length of the infinite system is $\xi_{\infty} \gg \Lambda^{\frac{1}{d}}$ :

$$
n_{b} \sim \Lambda^{-\frac{\beta}{v d}} \quad ; \quad c \sim \Lambda^{\frac{\alpha}{v d}} \quad ; \quad \chi \sim \Lambda^{\frac{\gamma}{v d}}
$$

where the exponents $(\alpha, \beta, \gamma)$ correspond to the behaviour of the infinite system: $n_{b} \sim t^{\beta} ; c \sim$ $t^{-\alpha} ; \chi \sim t^{-\gamma}$. In particular, the finite-size power law $n_{b} \sim \Lambda^{-\frac{\beta}{v d}}$ describes a geometrical structure of the system with fractal dimension which is accessible to observation through the phenomenon of intermittency: $\left\langle N_{b}\right\rangle \sim \Lambda^{q}$. It turns out that the fractal dimension of the critical system described by a $q$-partition function is $d_{F}=q d$. In the Ising-QCD system $\left(q=\frac{5}{6}, d=3\right)$ the fractal dimension is $d_{F}=\frac{5}{2}$ and gives a measure of critical fluctuations. Thus, the Ising-QCD partition function (3.6) provides us with a valuable effective description of critical QCD since it satisfies all the requirements of scaling and universality near the QCD critical point.

\section{Higher cumulants versus measurements at BNL-RHIC}

Higher cumulants of net-baryon multiplicity and in particular non-Gaussian kurtosis $\left(\kappa_{n G}\right)$ of net protons is widely used in the search for the critical point [13]. They are defined as:

$$
\kappa_{n G}=\frac{C_{4}-3 C_{2}^{2}}{C_{2}^{2}} \quad ; \quad C_{k}=\left\langle(N-\langle N\rangle)^{k}\right\rangle, k=2,3, . .
$$

It is argued that $\kappa_{n G}$ possesses a non-monotonic behaviour, attaining a negative minimum when crossing the critical point $[6,14]$.

The proposed Ising-QCD partition function leads to a prediction of $\kappa_{n G}$ along the two directions $(\ln \zeta, t)$ in the phase diagram, employing the relations:

$$
C_{2}=\frac{\partial^{2} \ln \mathscr{L}_{I Q C D}}{\partial(\ln \zeta)^{2}} ; \quad C_{4}-3 C_{2}^{2}=\frac{\partial^{4} \ln \mathscr{Z}_{I Q C D}}{\partial(\ln \zeta)^{4}}
$$

In Figure 3 we show the behaviour of the calculated kurtosis as a function of $\ln \zeta$ assuming $T=T_{c}$. A sharp minimum near $\mu=\mu_{c}$ is predicted as a signal for the critical point.

It is remarkable that the critical behaviour of the kurtosis cannot be captured in BES I measurements (RHIC-STAR) as illustrated in Fig. 4.

In fact the broad minimum suggested by the data is not linked to the QCD critical point. From theoretical point of view, the sharpness of the predicted minimum with a width of $\approx 5 \mathrm{MeV}$ is a manifestation of the narrowness of the critical region along the chemical potential direction. This can be demonstrated in a detailed study of the Ising-QCD partition function. The basic argument is that critical fluctuations are confined in a region of the phase diagram, around the critical point, where a geometrical power law holds: $\left\langle N_{b}\right\rangle \sim \Lambda^{\tilde{q}}$ with $\frac{3}{4}<\tilde{q}<1$. In the upper limit $(\tilde{q}=1)$, critical 


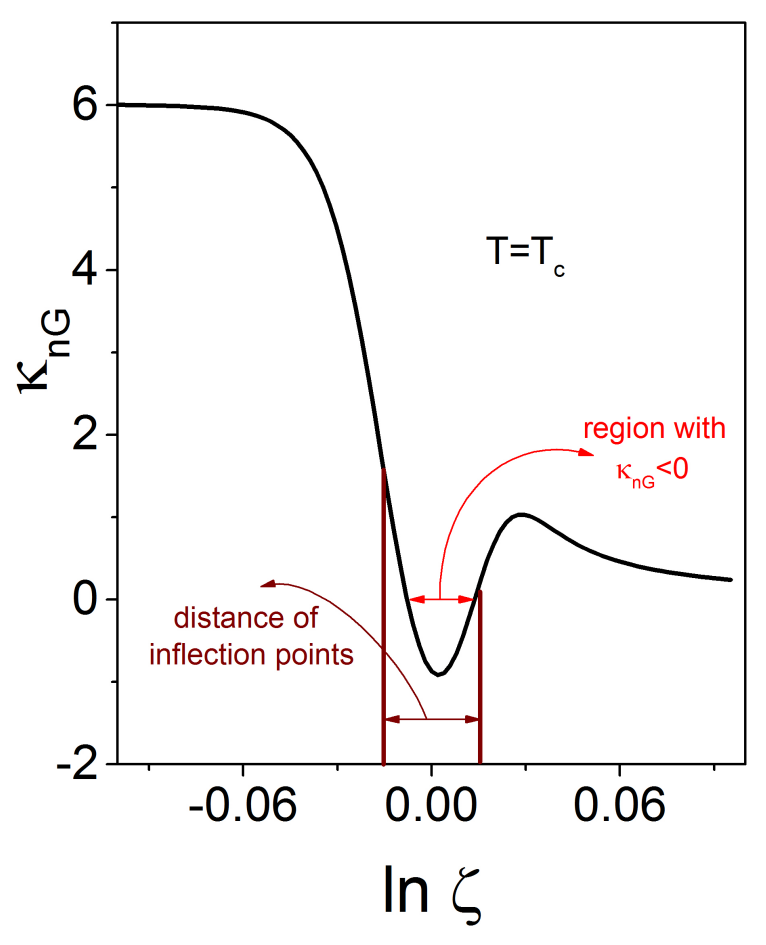

Figure 3: The non-Gaussian kurtosis $\kappa_{n G}$, calculated through the partition function $\mathscr{Z}_{I Q C D}$, as a function of $\ln \zeta$ for $T=T_{c}$.

fluctuations disappear whereas in the lower limit $\left(\tilde{q}=\frac{3}{4}\right)$ the fluctuations change universality class $\left(\phi^{6} \rightarrow \phi^{4}\right)$ and cannot be linked to the QCD critical point any more. The critical region is indeed narrow (see Fig. 5) with size, for $T=T_{c}, \Delta \mu \approx 5-7 \mathrm{MeV}$, compatible with the width of the sharp minimum of kurtosis.

\section{Critical fluctuations of proton density versus measurements at CERN-SPS}

According to our general discussion, the FSS property: $\left\langle N_{b}\right\rangle \sim \Lambda^{d_{F} / 3}$, implies a fractal structure of critical fluctuations at large scales: $\left\langle n(\vec{x}) n\left(\vec{x}^{\prime}\right)\right\rangle \sim\left|\vec{x}-\vec{x}^{\prime}\right|^{d_{F}-3}$ for $\left|\vec{x}-\vec{x}^{\prime}\right|=O\left(\Lambda^{1 / 3}\right)$. This singular behaviour is transferred to the density-density correlation in proton transverse momentum space for small momentum differences [15]: $\left\langle n(\vec{k}) n\left(\vec{k}^{\prime}\right)\right\rangle \sim\left|\vec{k}-\vec{k}^{\prime}\right|^{-\frac{2 d_{F}}{3}}\left(d_{F}=\frac{5}{2}\right)$. Such a singularity is detectable through intermittency studies in proton transverse momentum space [5]. The second 


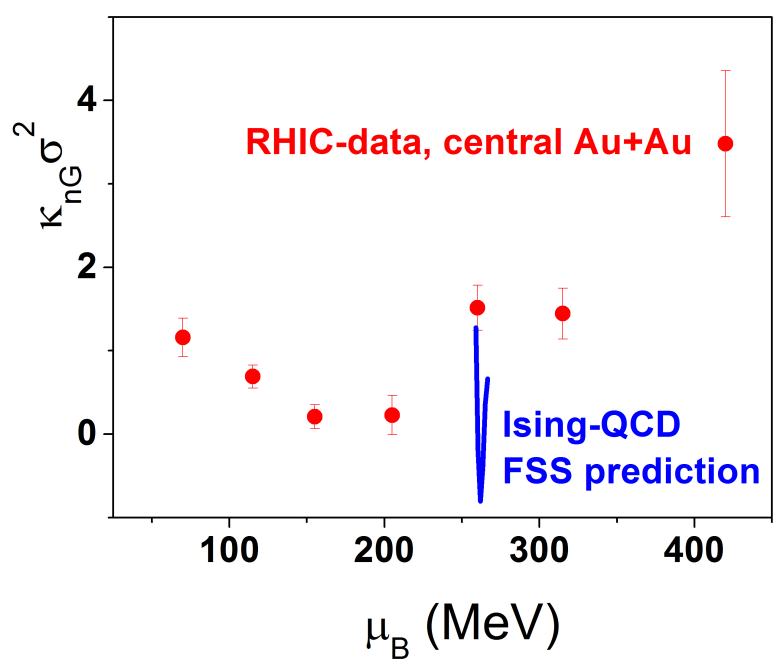

Figure 4: The non-Gaussian kurtosis $\kappa_{n G}$ (red circles) measured in central $\mathrm{Au}+\mathrm{Au}$ collisions at BES I (RHIC-STAR). The blue line is the result of the calculation of $\kappa_{n G}$ employing the Ising-QCD partition function.

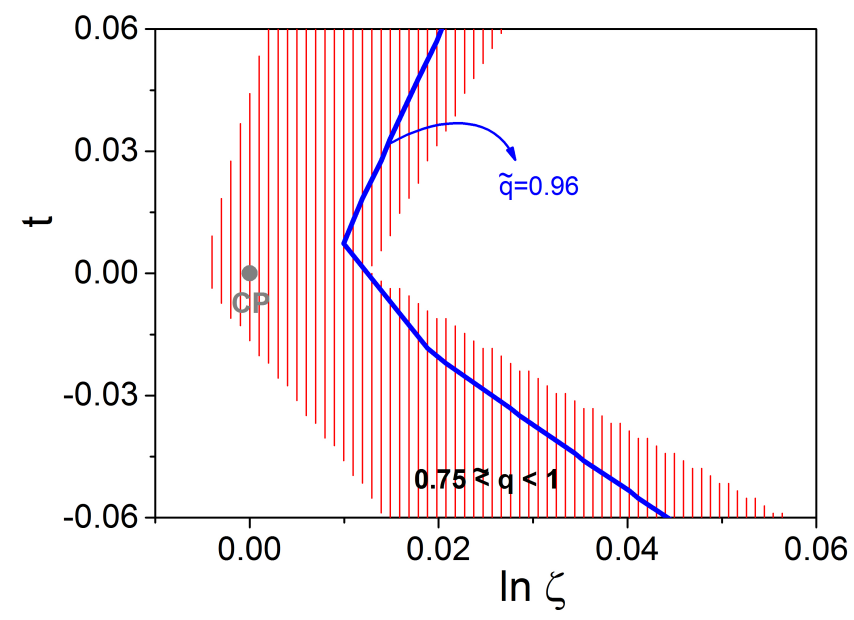

Figure 5: A map of the critical region in the $(\ln \zeta, t)$-plane, obtained through the Ising-QCD partition function. In the shaded region the scaling $\left\langle N_{b}\right\rangle \sim \Lambda^{\tilde{q}}$ with $\frac{3}{4}<\tilde{q}<1$ is valid.

factorial moment $F_{2}(M)$ in a cell of the transverse momentum space with side $\frac{1}{M}$ is expected to follow a power law of the form: $F_{2}(M) \sim M^{2 \phi_{2}}$ for large $M$ with a characteristic critical index $\phi_{2}=\frac{5}{6}$. Measurements at CERN-SPS have uncovered such a behaviour, but with large experimental uncertainties, in $\mathrm{Si}+\mathrm{Si}$ collisions at $158 \mathrm{GeV} / \mathrm{c}$ (NA49 experiment, [16]). The central value of the corresponding intermittency index was found $\tilde{\phi}_{2} \approx 0.96$, suggesting that the $\mathrm{Si}+\mathrm{Si}$ freeze-out state is located within the critical region, close to the boundary. This result may serve as a reference in order to proceed to a more detailed phenomenology of the critical point based on the Ising-QCD 


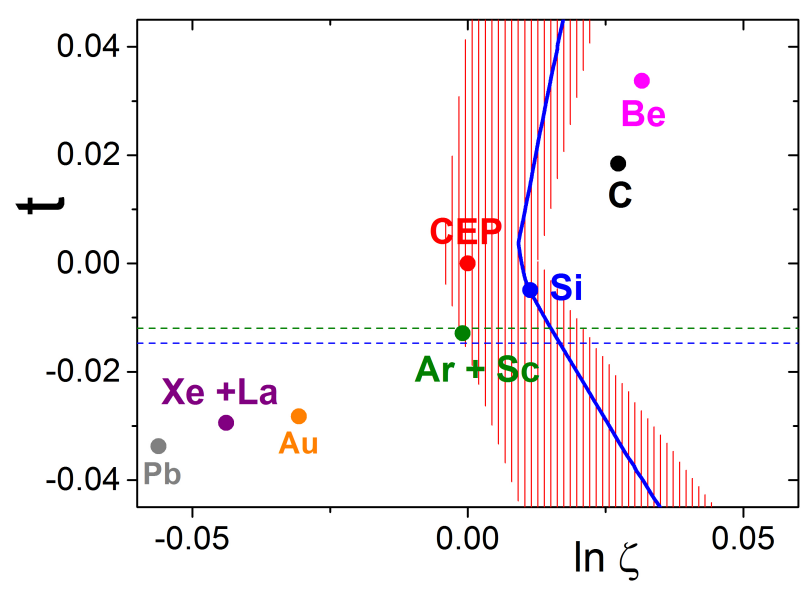

Figure 6: A map of the critical region in the $(\ln \zeta, t)$-plane containing the location of the measured freezeout states in NA49 experiment as well as predictions concerning the $\mathrm{Be}+\mathrm{Be}, \mathrm{Xe}+\mathrm{La}$ and $\mathrm{Ar}+\mathrm{Sc}$ freeze-out states expected to be measured in NA61/SHINE experiment. For completeness, we present also the Au + $\mathrm{Au}$ freeze-out state (central collisions) at $\sqrt{s}=14.5 \mathrm{GeV}$ (RHIC-STAR).

partition function and on new measurements at CERN-SPS.

In practice, the constraints imposed by FSS, Ising universality, recent measurements of freezeout states and intermittency measurements in $\mathrm{Si}+\mathrm{Si}, \mathrm{Pb}+\mathrm{Pb}$ and $\mathrm{C}+\mathrm{C}$ collisions at CERN-SPS (NA49) lead to the location of various systems in the phase diagram, showing their distance from the area of the critical region, as shown in Fig. 6. Only the process $\mathrm{Si}+\mathrm{Si}$ freezes out within the critical region while the freeze-out state of peripheral collisions in the Ar $+\mathrm{Sc}$ system may approach the boundary. Proton-intermittency analysis in this process is, at present, in progress.

\section{Summary}

It is demonstrated that the Ising-QCD universality leads to the construction of partition function which describes the QCD system (net baryons) close to the critical point. This description satisfies all requirements of scaling theory and universality. Based on this Ising-QCD partition function one can formulate predictions which are relevant to measurements in the search for the QCD critical point:

- The system develops a fractal structure at the critical point with dimension $d_{F}=\frac{5}{2}$, which is a measure of critical fluctuations. This fractal structure in configuration space implies selfsimilar fluctuations in transverse momentum space, detectable through intermittency analysis.

- The critical region is narrow along the chemical potential direction $(\Delta \mu \approx 5-7 \mathrm{MeV})$.

- A sharp minimum of kurtosis appears close to the critical point. The sharpness of this minimum is linked to the narrowness of the critical region. 
These predictions do not depend on the details of the description but only on the principles of Ising-QCD universality and FSS. The BES I measurements (RHIC-STAR) of net-proton kurtosis cannot capture the sharp minimum in the search for the critical point. The observed broad minimum is not related to the CEP. Measurements at CERN-SPS provide us with indications of critical fluctuations in the freeze-out states of $\mathrm{Si}+\mathrm{Si}$ central collisions at $158 \mathrm{~A} \mathrm{GeV} / \mathrm{c}$ (NA49). This opens up the perspective to detect critical fluctuations in peripheral $\mathrm{Ar}+\mathrm{Sc}$ collisions at $150 \mathrm{~A} \mathrm{GeV/c}$ (NA61/SHINE).

\section{References}

[1] Y. Aoki, et al., Nature 443, 675 (2006); T. Bhattacharya, et al., Phys. Rev. Lett. 113, 082001 (2014); P. Steinbrecher (for the HotQCD Collaboration), Nucl. Phys. A 982, 847 (2019).

[2] H. R. Jaqaman, A. Z. Mekjian and L. Zamick, Phys. Rev. C 29, 2067 (1984); M. A. Halasz, A. D. Jackson, R. E. Shrock, M. A. Stephanov and J. J. M. Verbaarschot, Phys. Rev. D 58, 096007 (1998); J. Berges and K. Rajagopal, Nucl. Phys. B538, 215 (1999).

[3] M. Buballa, Phys. Rept. 407, 205 (2005).

[4] Y. Hatta and M. A. Stephanov, Phys. Rev. Lett. 91, 102003 (2003).

[5] N. G. Antoniou, F. K. Diakonos, A. S. Kapoyannis and K. S. Kousouris, Phys. Rev. Lett. 97, 032002 (2006)

[6] M. A. Stephanov, Phys. Rev. Lett. 107, 052301 (2011).

[7] R. Pisarski and F. Wilczek, Phys. Rev. D 29, 338 (1984).

[8] Y. Hatta and T. Ikeda, Phys. Rev. D 67, 014028 (2003).

[9] P. Gerber, H. Leutwyler, Nucl. Phys. B 321, 387 (1989); T. D. Cohen, R. J. Furnstahl, D. K. Griegel, Phys. Rev. C 45, 1881 (1992).

[10] M. M. Tsypin, Phys. Rev. Lett. 73, 2015 (1994).

[11] N. G. Antoniou, F. K. Diakonos, X. Maintas and C. E. Tsagkarakis, Phys. Rev. D 97, 034015 (2018).

[12] C. N. Yang and T. D. Lee, Phys. Rev. 87, 404 (1952); T. D. Lee and C. N. Yang, Phys. Rev. 87, 410 (1952).

[13] X. Luo and N. Xu, Nucl. Sci. Tech. 28, 112 (2017); arXiv:1701.02105 [nucl-ex].

[14] N. G. Antoniou, F. K. Diakonos, N. Kalntis and A. Kanargias, Nucl. Phys. A 986, 167 (2019).

[15] N. G. Antoniou and F. K. Diakonos, J. Phys. G: Nucl. Part. Phys. 46, 035101 (2019).

[16] T. Anticic et al., Eur. Phys. J. C 75, 587 (2015). 